\title{
高锰酸钾法测定白云石中钻的影响机制探究
}

向思佳 ${ }^{1}$, 廖光旭 ${ }^{1}$, 姚奇志 $1,2,{ }^{*}$, 金谷 ${ }^{1}$, 李玲玲 1,2

1 中国科学技术大学化学与材料科学学院, 合肥 230026

2 化学国家级实验教学示范中心(中国科学技术大学), 合肥 230026

摘要: “白云石中钻含量测定” 是大学分析化学实验中重要的实验内容之一, 学生在进行该实验时发现, 用草酸钠标准 溶液标定高锰酸钾溶液和用标定过的高锰酸钾溶液滴定由白云石转化形成的草酸钙时, 其起始氧化还原反应速度存在明 显的差异。针对这些实验现象, 结合理论知识, 提出了几点猜想, 并指导学生设计实验进行探究和验证。通过这些实验 探究, 不仅解答了学生的疑惑, 更重要的是培养和提高了他们分析和解决问题的能力, 激发了学生对化学实验学习的兴 趣。

关键词: 高锰酸钾法; 氧化还原反应; 草酸钙; 影响因素; 滴定速度

中图分类号: G64; O6

\section{A Study on Influence Mechanism in Determination of Calcium Content in Dolomite by Potassium Permanganate Titration}

\author{
Sijia Xiang ${ }^{1}$, Guangxu Liao ${ }^{1}$, Qizhi Yao ${ }^{1,2, *}$, Gu Jin ${ }^{1}$, Lingling Li ${ }^{1,2}$ \\ ${ }^{1}$ School of Chemistry and Materials Science, University of Science and Technology of China, Hefei 230026, China. \\ ${ }^{2}$ National Demonstration Center for Experimental Chemistry Education (University of Science and Technology of China), \\ Hefei 230026, China.
}

\begin{abstract}
Determination of calcium content in dolomite" is one of the important experiments in college analytical chemistry laboratory. During the experiment, students found that the initial redox reaction rates are significantly different between the calibration experiment of potassium permanganate solution by sodium oxalate standard solution and the titration experiment of calcium oxalate formed from dolomite by the calibrated potassium permanganate solution. Based on these experimental phenomena and combined with chemistry theory mastered by students, some conjectures and explanations were put forward. After that, we guided students to design experiments to explore and verify them. Through these experiments, it can not only answer the students' doubts, but also train and improve their ability to analyze and solve problems, and thus stimulating students' interest in chemical experiment learning.
\end{abstract}

Key Words: Potassium permanganate method; Redox reaction; Calcium oxalate; Influence factors; Titration rate.

高锰酸钾是一种强氧化剂, 利用高锰酸钾的强氧化能力发展形成的高锰酸钾滴定法, 不仅可在 不同 $\mathrm{pH}$ 下测定多种无机物和有机物的含量, 而且 $\mathrm{MnO}_{4}^{-}$本身具有特殊的紫红色, 可避免滴定过程中 指示剂的添加; 同时, 其氧化还原反应往往是自催化的。因此, 高锰酸钾滴定法是氧化还原滴定中 
最经典的滴定分析之一。其缺点是高锰酸钾溶液不太稳定; $\mathrm{Mn}$ 的多种价态决定了其氧化还原反应历 程较复杂, 易于发生不利于测定的副反应。但如果高锰酸钾溶液配制和保存方法得当, 滴定时严格 控制酸碱度、温度、滴定速度等实验条件, 可大幅度减少副反应的影响 ${ }^{[1]}$, 达到测定的精度和准确度 要求。“白云石中钙含量测定” 就是利用高锰酸钾滴定由白云石 $\left[\mathrm{CaMg}\left(\mathrm{CO}_{3}\right)_{2}\right]$ 转化形成的草酸钙 $\left(\mathrm{CaC}_{2} \mathrm{O}_{4}\right)$, 间接测定矿物白云石中钙含量。其主要过程为: 首先用 $\mathrm{HCl}$ 溶液溶解白云石矿样, 再用沉 淀剂 $\left(\mathrm{NH}_{4}\right)_{2} \mathrm{C}_{2} \mathrm{O}_{4}$ 溶液将 $\mathrm{Ca}^{2+}$ 完全沉淀为草酸钙, 过滤洗涤得到草酸钲沉淀, 将带有沉淀的滤纸贴在烧 杯壁上, 用热蒸馏水将滤纸上的大部分沉淀洗入烧杯中, 再用 $\mathrm{H}_{2} \mathrm{SO}_{4}$ 溶液溶解沉淀并控制酸度, 趁热 用高锰酸钾标准溶液滴定。高锰酸钾溶液浓度用草酸钠基准标定 ${ }^{[2]}$ 。该实验要求滴定开始时温度控 制在 $70-80{ }^{\circ} \mathrm{C}$, 滴定结束温度不低于 $60^{\circ} \mathrm{C}$; 滴定起始酸碱度控制在 $\left[\mathrm{H}^{+}\right]=1 \mathrm{moL} \cdot \mathrm{L}^{-1}$; 滴定速度为 慢-快-慢, 即滴定开始时, 必须待前一滴粉红色完全褪去才能滴下一滴, 滴定速度随裉色速度加快而 加快, 近终点时要求逐滴加入, 溶液颜色突变为粉红色半分钟不裉去, 达到终点。

该实验成功的关键是草酸钙沉淀的制备和滴定条件的严格控制。要求制备的草酸钲沉淀晶体颗 粒大而且均匀, 避免吸附过多杂质引起误差; 滴定开始控制好酸碱度、温度和滴定速度, 结果误差 可以控制在 $3 \%$ 以内。然而, 学生在实验过程中, 经常会发现两种明显存在的实验现象: 1) 在样品 测定中, 用高锰酸钾溶液滴定草酸钙溶液与标定过程中用高锰酸钾滴定草酸钠溶液对比时, 发现前 者在滴入第一滴高锰酸钾溶液后的褪色速率显著大于后者; 2) 用草酸钠标定高锰酸钾时, 第一滴高 锰酸钾溶液颜色常常需要 5-20 min才能褪色; 但部分学生不小心直接加入了数滴乃至 $1 \mathrm{~mL}$ 高锰酸钾 溶液时, 高锰酸钾的粉红色往往会在几十秒内褪色。

针对第一个现象, 笔者对比分析了标定与滴定反应时的实验条件差异, 提出了几点猜想: (1) 滴 定过程与标定过程存在金属离子的差异: 标定的基准物质是草酸钠, 溶液中有 $\mathrm{Na}^{+}$; 滴定过程中, 被 滴定物是草酸钙, 溶液中有 $\mathrm{Ca}^{2+}$; 由此推测溶液中 $\mathrm{Ca}^{2+}$ 可能催化了反应的快速发生, 使得第一滴高锰 酸钾溶液的粉红色裖色加快。(2) 滴定过程与标定过程存在滤纸的差异: 标定的基准物质是草酸钠, 被滴定物只有草酸钠; 滴定过程中, 除了被测物质草酸钙, 还有用于过滤分离草酸钙沉淀的滤纸存 在; 因此, 推测滤纸可能催化了反应的进行。(3) 滴定过程中的滤纸可能充当还原剂: 过滤用的普通 滤纸主要由棉花纤维制成, 滤纸中可能存在少量的还原性物质, 其还原能力可能比 $\mathrm{C}_{2} \mathrm{O}_{4}^{2-}$ 更强, 优先 与高锰酸钾发生氧化还原反应生成 $\mathrm{Mn}^{2+}, \mathrm{Mn}^{2+}$ 进一步催化草酸根与高锰酸根的反应。后两种假设虽 然都与滤纸有关, 但其机理不同, 结果也不同。假设(2)中滤纸只作为催化剂, 则对滴定终点无影响; 而假设(3)滤纸中的成分发挥了还原剂的作用, 会对滴定终点产生影响。鉴于此, 我们分别设计不同 条件实验进行实验探究, 试图阐明上述实验现象背后蕴藏的化学反应过程和本质。

\section{1 验证实验}

\section{1 实验试剂}

草酸钠(AR), 高锰酸钾(AR), 硫酸(AR), 碳酸钙(AR), 所有试剂均购自国药集团。

\section{2 溶液的配制}

1) $0.05 \mathrm{~mol} \cdot \mathrm{L}^{-1}$ 草酸钠标准溶液: 用分析天平称取 $3.3500 \mathrm{~g}$ 分析纯的草酸钠, 用蒸馏水定容至 $500 \mathrm{~mL}$ 容量瓶中, 得到 $0.05000 \mathrm{~mol} \cdot \mathrm{L}^{-1}$ 的草酸钠标准溶液。

2) $0.02 \mathrm{~mol} \cdot \mathrm{L}^{-1}$ 高锰酸钾溶液: 量取 $60 \mathrm{~mL} 0.1 \mathrm{~mol} \cdot \mathrm{L}^{-1}$ 高锰酸钾溶液, 在试剂瓶中稀释到 $300 \mathrm{~mL}$, 摇匀, 备用。

\section{3 高锰酸钾溶液浓度的标定}

准确移取 $25.00 \mathrm{~mL}$ 草酸钠标准溶液至 $250 \mathrm{~mL}$ 烧杯中, 加入 $120 \mathrm{~mL}$ 热蒸馏水, 加热至近沸, 加入 体积比为 $1: 2$ 的 $\mathrm{H}_{2} \mathrm{SO}_{4}$ 溶液 $10 \mathrm{~mL}$, 立即用高锰酸钾溶液滴定至粉红色, 且半分钟不裉色, 记录消耗 的高锰酸钾溶液体积。滴定开始需规范确保每一滴粉红色褪去再滴加下一滴, 滴定过程需用玻璃棒 摚动溶液。 


\section{4 高锰酸钾溶液快滴的探究}

与高锰酸钾溶液浓度标定相同, 但最初不是逐滴加入, 分别直接快速滴加 $1 \mathrm{~mL}$ 和 $5 \mathrm{~mL}$ 高锰酸钾 溶液。

\section{5 对金属离子差异的探究}

与高锰酸钾溶液浓度标定相同, 但在滴定前加入 $0.12 \mathrm{~g}$ 碳酸钻。

\section{6 滤纸存在差异的探究}

用草酸钠标定高锰酸钾溶液浓度过程没有滤纸, 而用高锰酸钾溶液滴定草酸钙的过程存在着过 滤用的滤纸, 与高锰酸钾溶液浓度标定相同, 但在滴定前贴壁分别加入一张、半张与过滤用同批次 滤纸。

\section{7 温度条件实验}

该实验要求滴定时, 溶液温度控制在 $70-80^{\circ} \mathrm{C}$, 滴定结束时温度不能低于 $60^{\circ} \mathrm{C}$ 。在实验过程中, 几乎相同的操作, 高锰酸钾褪色速度也会存在差异, 推测是因为滴定开始温度差异的影响, 为了探 究温度对实验的影响, 添加了室温下直接滴定实验组, 观察实验现象并记录第一滴溶液褪色所需时间。

\section{8 实验数据记录}

以上实验得到的数据分别记录在表 1 和表 2 中。

表1 不同滴定条件下高锰酸钾溶液的褪色时间

\begin{tabular}{lcccc}
\hline \multicolumn{1}{c}{ 滴定数据 } & 第一组 & 第二组 & 第三组 & 第四组 \\
\hline 第一次溶液褪色所需时间 & $7 \mathrm{~min} 30 \mathrm{~s}$ & $20 \mathrm{~s}$ & $1 \mathrm{~min}$ & $19 \mathrm{~min}$ \\
滴至终点消耗体积 & $35.30 \mathrm{~mL}$ & - & - & - \\
\hline
\end{tabular}

第一组为标定, 第二、三组分别加入一张滤纸和半张滤纸, $70-80^{\circ} \mathrm{C}$; 第四组是室温下的滴定

表2 不同滴定条件下高锰酸钾溶液褪色时间和消耗体积

\begin{tabular}{lcccc}
\hline 滴定数据 & 第一组 & 第二组 & 第三组 & 第四组 \\
\hline 第一次溶液褪色所需时间 & $30 \mathrm{~s}$ & $40 \mathrm{~s}$ & $18 \mathrm{~s}$ & $5 \mathrm{~s}$ \\
滴至终点消耗体积 & $35.45 \mathrm{~mL}$ & $35.41 \mathrm{~mL}$ & $35.40 \mathrm{~mL}$ & $35.39 \mathrm{~mL}$ \\
\hline 滴定数据 & 第五组 & 第六组 & 第七组 & 第八组 \\
\hline 第一滴溶液褪色所需时间 & $8 \operatorname{min~} 30 \mathrm{~s}$ & $8 \mathrm{~min}$ & $1 \mathrm{~min} 30 \mathrm{~s}$ & $30 \mathrm{~s}$ \\
滴至终点消耗体积 & $35.30 \mathrm{~mL}$ & $35.38 \mathrm{~mL}$ & $35.58 \mathrm{~mL}$ & $35.60 \mathrm{~mL}$ \\
\hline
\end{tabular}

第一、二组为 $1 \mathrm{~mL}$ 组, 第三、四组为 $5 \mathrm{~mL}$ 组, 第五、六组为加钻组, 第七、八组为加滤纸组, $70-80{ }^{\circ} \mathrm{C}$

\section{2 结果与讨论}

我们发现严格按照教材规范操作进行标定实验, 滴定开始溶液温度为 $80^{\circ} \mathrm{C}$ 时, 第一滴高锰酸钾 粉红色完全褪去需要 $7 \mathrm{~min} 30 \mathrm{~s}$, 滴定终点消耗高锰酸钾溶液体积为 $35.30 \mathrm{~mL}$ (表1)。若实验开始时迅 速加入 $1 \mathrm{~mL}$ 和 $5 \mathrm{~mL}$ 高锰酸钾溶液进行快滴, 我们发现此时高锰酸钾能迅速与草酸反应, 在几秒到几 十秒内就褪色。并且在快速加入 $5 \mathrm{~mL}$ 高锰酸钾的实验组中, 还观察到大量棕褐色沉淀产生, 推测是 未来得及与草酸反应的高锰酸钾自分解生成的二氧化锰。快滴组终点消耗高锰酸钾溶液平均体积分 别为 $35.43 \mathrm{~mL}$ 和 $35.40 \mathrm{~mL}$ (表2), 比标定实验组的 $35.30 \mathrm{~mL}$ 多消耗高锰酸钾溶液体积约 $0.1 \mathrm{~mL}$, 在其 他条件均相同的情况下, 利用假设检验法, 最终计算得到统计量的观察值 $t=8.555>t_{0.05}(3)=2.353^{[3]}$, 指示在 $95 \%$ 的置信度下, 快滴组平均消耗高锰酸钾溶液显著高于标定组。这说明, 尽管慢滴的反应 时间更长, 但快滴时更高的高镇酸钾浓度会导致更多的高锰酸钾自分解。这是因为高锰酸钾在强酸 性条件下发生自分解反应而放出氧气, 其反应方程式如下:

$$
4 \mathrm{MnO}_{4}^{-}+12 \mathrm{H}^{+} \rightarrow 4 \mathrm{Mn}^{2+}+5 \mathrm{O}_{2}+6 \mathrm{H}_{2} \mathrm{O}^{[4]}
$$


而这多消耗的约 $0.1 \mathrm{~mL}$ 高锰酸钾溶液, 导致近 $3 \%$ 的误差, 在定量分析中这是不允许的。这也就 是为什么高锰酸钾法要求滴定速度慢-快-慢, 即开始逐滴加入, 等粉红色裉去才能加入下一滴的原因。

对比表 2 第一至第四组的数据发现, 快滴 $5 \mathrm{~mL}$ 比快滴 $1 \mathrm{~mL}$ 褪色更快, 因为快滴 $5 \mathrm{~mL}$ 产生更多的 $\mathrm{Mn}^{2+}$ 起到了催化剂作用, 导致反应速度更快。此外, 在直接快速放入 $5 \mathrm{~mL}$ 高锰酸钾的快滴实验中, 起初产生了大量棕褐色沉淀, 随着滴定继续进行, 棕色沉淀在几十秒内逐渐消失, 而且终点消耗的 高锰酸钾溶液体积没有同等增多, 推测产生的棕色沉淀二氧化锰与草酸钠发生进一步反应, 最终生 成二价锰离子, 该推测在马文清等 ${ }^{[5]}$ “草酸钠-高锰酸钾返滴定法快速测定软锰矿中的 $\mathrm{MnO}_{2}$ ” 得到佐 证, 即滴定中途产生的二氧化锰最终与草酸钠反应生成二价锰, 导致消耗的高锰酸钾溶液体积没有 同步增大。

金属离子差异的影响: 标定的基准物质是草酸钠, 溶液中有 $\mathrm{Na}^{+}$; 但滴定过程中被滴定物是草酸 钙, 溶液中存在 $\mathrm{Ca}^{2+}$; 由此推测溶液中 $\mathrm{Ca}^{2+}$ 可能催化了反应的快速发生, 使得第一滴高锰酸钾溶液的 粉红色褪色加快。而 $\mathrm{Ca}^{2+}$ 是非变价的碱土金属离子, 其作为氧化还原催化剂的可能性几乎没有, 所 以从化学原理上说该推测不成立。实验结果也进一步给出证实。根据加钙组的数据与标定数据对比 可知, 单独加入钙离子对反应速率的影响并不大, 终点消耗高锰酸钾溶液体积没有明显差别(表2), 因此，钙离子对滴定开始的反应速度和终点消耗体积没有影响，这与理论分析完全吻合。

滤纸存在的影响: 对加入滤纸组与标定对照组数据(表1、2)进行分析, 发现正是滤纸的加入加速 了反应速度。同时, 滤纸组比标定组的终点高锰酸钾消耗量多出 $0.30 \mathrm{~mL}$ 左右, 说明滤纸上的确有还 原性物质与高锰酸钾反应。因此实验操作中要求 “将滤纸贴壁, 等褪色后再将滤纸全部推入溶液” 是必要的。若最初就将滤纸浸入, 其中的还原性物质会充分和高锰酸钾溶液反应, 造成更大误差; 为此我们设置了加入一整张滤纸和半张滤纸的对比实验 (表1), 实验表明第一滴溶液褪色时间和加入 滤纸的量有关, 这进一步证明滤纸上的还原性物质加速了初始反应速度, 导致高锰酸钾粉红色褪色 加快。

滴定起始温度影响: 在实验过程中, 发现温度差异对高锰酸钾与草酸钠的反应有明显影响, 为 此, 开展不同温度的滴定实验。表1列出了室温下高锰酸钾溶液滴定草酸钠的实验结果, 发现第一滴 高锰酸钾溶液的粉红色裉色用了近 $20 \mathrm{~min}$, 而且实验过程中还观察到溶液颜色呈现一系列改变, 即 经历了由紫红 $\rightarrow$ 桃红 $\rightarrow$ 橙红 $\rightarrow$ 黄色, 最后到无色的复杂过程, 实验最终消耗的高锰酸钾体积误差也 很大。因此, 温度对高钽酸钾滴定法影响较大, 控制滴定初始温度为 $80^{\circ} \mathrm{C}$ 左右, 滴定结束温度不低 于 $60^{\circ} \mathrm{C}$ 是很必要的。

\section{3 结语}

探究实验结果表明, 快滴会使高锰酸钾溶液发生自分解从而带来一定的负误差; 钻离子对滴定 速度没有影响; 滤纸中的还原性物质可以快速与高锰酸钾反应生成锰离子, 这些锰离子又作为催化 剂催化反应进行, 从而使高锰酸钾粉红色褪色加快。但滤纸的浸入也会带来一定的误差, 不同批次 的滤纸含有的还原性物质量不等, 实验统计显示存在 $0.05-0.30 \mathrm{~mL}$ 左右的负误差, 因此, 对于白云石 中钙含量测定的实验, 实验步骤中强调先慢后快再慢是有必要的, 同时也建议添加同批次滤纸空白 实验, 以提高实验的准确度。

\section{参 考 文 献}

[1] 李龙泉, 林长山, 朱玉瑞, 吕敬慈, 江万权. 定量化学分析. 合肥: 中国科学技术大学出版社, 1997: 193.

[2] 金谷, 姚奇志, 江万权, 胡祥余, 李娇. 分析化学实验. 合肥: 中国科学技术大学出版社, 2010: 119 .

[3] 邵利民. 分析化学. 北京: 科学出版社, 2016: 43-44.

[4] 李龙泉, 林长山, 朱玉瑞, 吕敬慈, 江万权. 定量化学分析. 合肥: 中国科学技术大学出版社, 1997: 194.

[5] 马清文, 王俊秀, 李艳丽, 李龙霞, 国红杰. 山东冶金, 2009, 31 (5), 150. 